\title{
Wake patterns of the wings and tail of hovering hummingbirds
}

\author{
Douglas L. Altshuler · Marko Princevac • \\ Hansheng Pan · Jesse Lozano
}

Received: 17 June 2008/Revised: 17 November 2008/Accepted: 28 November 2008/Published online: 28 December 2008

(C) The Author(s) 2008. This article is published with open access at Springerlink.com

\begin{abstract}
The flow fields of slowly flying bats and fasterflying birds differ in that bats produce two vortex loops during each stroke, one per wing, and birds produce a single vortex loop per stroke. In addition, the circulation at stroke transition approaches zero in bats but remains strong in birds. It is unknown if these difference derive from fundamental differences in wing morphology or are a consequence of flight speed. Here, we present an analysis of the horizontal flow field underneath hovering Anna's hummingbirds (Calypte anna) to describe the wake of a bird flying at zero forward velocity. We also consider how the hummingbird tail interacts with the wake generated by the wings. High-speed image recording and analysis from three orthogonal perspectives revealed that the wing tips reach peak velocities in the middle of each stroke and approach zero velocity at stroke transition. Hummingbirds use complex tail kinematic patterns ranging from in phase to antiphase cycling with respect to the wings, covering several phase shifted patterns. We employed particle image velocimetry to attain detailed horizontal flow measurements at three levels with respect to the tail: in the tail, at the tail tip, and just below the tail. The velocity patterns underneath the wings indicate that flow oscillates along the ventral-dorsal axis in response to the down- and up-strokes and that the sideways flows with respect to the bird are consistently from the lateral to medial. The region around the tail is dominated by axial flows in dorsal to ventral
\end{abstract}

D. L. Altshuler $(\bowtie)$

Department of Biology, University of California,

Riverside, CA 92521, USA

e-mail: douga@ucr.edu

M. Princevac $\cdot$ H. Pan · J. Lozano

Department of Mechanical Engineering,

University of California, Riverside, CA 92521, USA direction. We propose that these flows are generated by interaction between the wakes of the two wings at the end of the upstroke, and that the tail actively defects flows to generate moments that contribute to pitch stability. The flow fields images also revealed distinct vortex loops underneath each wing, which were generated during each stroke. From these data, we propose a model for the primary flow structures of hummingbirds that more strongly resembles the bat model. Thus, pairs of unconnected vortex loops may be shared features of different animals during hovering and slow forward flight.

\section{Introduction}

Since Kokshaysky (1979) first photographed birds flying through wood and paper dust clouds, flight biologists have been working to attain higher resolution views and quantifiable features of the wakes of flying animals. Experiments have focused on vertical measurement planes with one view or two orthogonal views to characterize the momentum imparted behind and below the animal (Spedding 1986, 1987a, b; Spedding et al. 1984). Recent efforts have yielded high-resolution, three-dimensional descriptions of the wakes produced during moderate and fast forward flight in passerine birds (Hedenström et al. 2006; Rosén et al. 2007; Spedding et al. 2003), and also during slow and moderate forward flight in bats, the only other extant vertebrate lineage that has powered, flapping flight (Hedenström et al. 2007; Muijres et al. 2008). In birds, the two wings together produce a single vortex loop, whereas in bats each wing produces a separate vortex loop. In addition, birds generate considerable circulation during stroke transitions whereas circulation during bat flapping 
approaches zero at stroke transition. It is unknown to what extent these alternative wake structures derive from differences in flight speed versus lineage-specific differences in the morphology and neuromuscular control of the wings.

The hovering flight of hummingbirds provides an excellent opportunity to examine the wake structure produced by birds during hovering and thus with no forward velocity. The flow fields generated by hovering rufous hummingbirds (Selasphorus rufus) have been described from two orthogonal, vertical perspectives with the results that the wings generate a powerful wake oriented directly below the animal, which is dominated by the much stronger downstroke (Warrick et al. 2005). A horizontal view of the wakes underneath hovering hummingbirds has not been described, but this perspective should provide unique information about the wing wake structures. A horizontal perspective has the additional advantage of providing pertinent information concerning how the hummingbird tail interacts with the very strong downwards wing wakes.

Three aerodynamic functions for bird tails are known. During forward flight tails (1) function in maneuverability and stability, (2) can help control the angle of attack of the wings, and (3) during slow forward flight can produce lift (Thomas 1997; Thomas and Balmford 1995). However, the elaborated tails of some species are elongated in response to sexual selection and increase costs of parasitic drag (Balmford et al. 1993; Evans and Hatchwell 1992; Møller 1989; Norberg 1995). The aerodynamic function of the tail in hovering flight is not clear given the absence of a translational flow velocity, but it has been suggested that the tail may be generally involved in pitch control (references in Warrick et al. 2002), which should be important for maintaining stability during hovering.

Here we describe the relative motion of the hummingbird wings and tail, and the horizontal wake pattern in a two-dimensional plane. We separately considered three elevations of perspective: a plane centered within the tail, a plane at the tail tip, and a plane located just below the tail. We present velocity profiles based upon temporal sequences of wing positions. These measurements provide information about the wake structure, the tail-wake interaction, and the horizontal orientation of the velocity vectors. We use these data to propose a model of the dominant flow structures produced during hovering flight in hummingbirds.

\section{Phase relationships between hummingbird wings and tail}

The wing and tail kinematics of hovering hummingbirds were studied using five male Anna's hummingbirds
(Calypte anna), which were captured in Berkeley (three birds) and Pasadena (two birds), California. These hummingbirds were housed in institutional vivaria and trained to feed from artificial feeders made from $10 \mathrm{ml}$ syringes. For filming trials, the hummingbirds were moved from the vivaria to an acrylic chamber $(1 \mathrm{~m} \times 1 \mathrm{~m} \times 1 \mathrm{~m})$ constructed with three clear sides and three opaque sides. The chamber contained a single perch and a single artificial feeder, also made from a 10-ml syringe. Hovering bouts were filmed from three perspectives (top and two sides) using three digital high-speed cameras filming at 1,000 frames per second. The Institutional Animal Care and Use Committees at the University of California Berkeley and the California Institute of Technology approved these vertebrate animal procedures.

One hovering sequence for each of the five birds was analyzed via frame-by-frame digitization with custom software (Fry et al. 2003) written in Matlab (Mathworks, Inc.). The three-dimensional coordinates of the wing and tail tips were assigned for five complete wingbeats for each bird. We calculated the vector velocity of the tips of the wings and tail based upon motion through the $x, y$, and $z$ planes. During hovering bouts, wingtip velocities were maximal during the middle of each stroke and approached 0 during stroke transitions. All five birds beat their tails in a harmonic motion, but two hummingbirds moved the tail in antiphase with the wings, one animal moved its tail in phase with the wings, and two animals exhibited a shift between phase and antiphase within the short sequence. An example of this latter category is provided in Fig. 1.

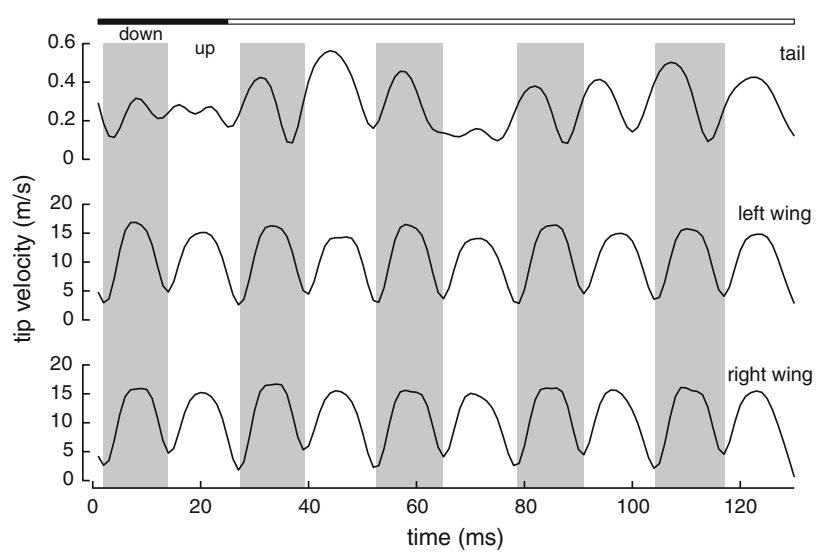

Fig. 1 The velocities of the tail and two wings of a hovering hummingbird. Five complete wingbeats are plotted with downstrokes indicated in gray and upstrokes in white. In the beginning of this sequence during the downstroke, the hummingbird moved its tail in antiphase (top black bar) to the wings, but used an extra tail cycle during the first upstroke to shift to in phase movement between the wings and the tail (top white bar) 


\section{Methods for recording flow features in hovering hummingbirds}

We measured the flow characteristics of three male Anna's hummingbirds ( $C$. anna), captured in Riverside, CA, USA and housed in the vivarium at the University of California Riverside (UCR). The Institutional Animal Care and Use Committee at UCR approved these vertebrate animal procedures.

Prior to each experiment, an individual bird was moved from the vivarium to an experimental flight chamber for 1 day of training in the new setting. The dimensions of the flight chamber were $76 \mathrm{~cm} \times 76 \mathrm{~cm} \times 95 \mathrm{~cm}$ high and it was constructed of a PVC frame with walls made of thin plastic sheeting (Fig. 2a). The chamber contained a single perch and one small feeder constructed from a 1-ml syringe. In addition to becoming accustomed to the flight chamber and feeder, we also trained the hummingbirds to feed on command by restricting access to the feeder for $15 \mathrm{~min}$ and then only allowing one feeding bout before again restricting access. A feeding bout was defined as a single episode of hovering at the feeder until the bird moved more than approximately two body lengths away in any direction.

Experiments began the day after training and involved the same feeding protocol. A particle image velocimetry (PIV) system was used to measure the instantaneous velocity field in the horizontal plane. The PIV technique measures the velocity in a fluid by correlating images of the particle-seeded flow (Adrian 1988). For these experiments, atomized food grade olive oil was utilized as the seeding particles. In the minute prior to providing feeder access, we filled the chamber with olive oil mist created using a pressurized oil container equipped with a perforated tube to enable oil-air mixing for adequate atomization.

As the bird approached the feeder, the olive oil particles were illuminated in a plane below the feeder using a horizontal laser sheet with a wavelength of $532 \mathrm{~nm}$ (with energy of $388 \mathrm{~mJ} /$ pulse) generated by a double-pulsed Nd:YAG laser (Big Sky Laser Technologies, Inc, model CFR400) located $0.5 \mathrm{~m}$ from the feeder. The Q-switch was set to $100 \mu$ s corresponding to approximately medium laser power. The laser beam was expanded into a $20^{\circ}$ diverging light sheet using sheet-forming optics, which included a cylindrical lens ( $-15 \mathrm{~mm}$ focal length) and a spherical lens (200 $\mathrm{mm}$ focal length). In this way, the beam was transformed into a $200-\mathrm{mm}$ wide and $0.212 \mathrm{~mm}$ thick sheet to illuminate seed particles in a horizontal plane close to the bird. In separate experiments, we illuminated three planes: (1) $1 \mathrm{~cm}$ above the middle plane, i.e., within the tail, (2) at the tail tip during its cyclical oscillation, and (3) just underneath the tail. A LASERPULSE Synchronizer (TSI Inc.) was utilized to trigger the laser pulse and the camera

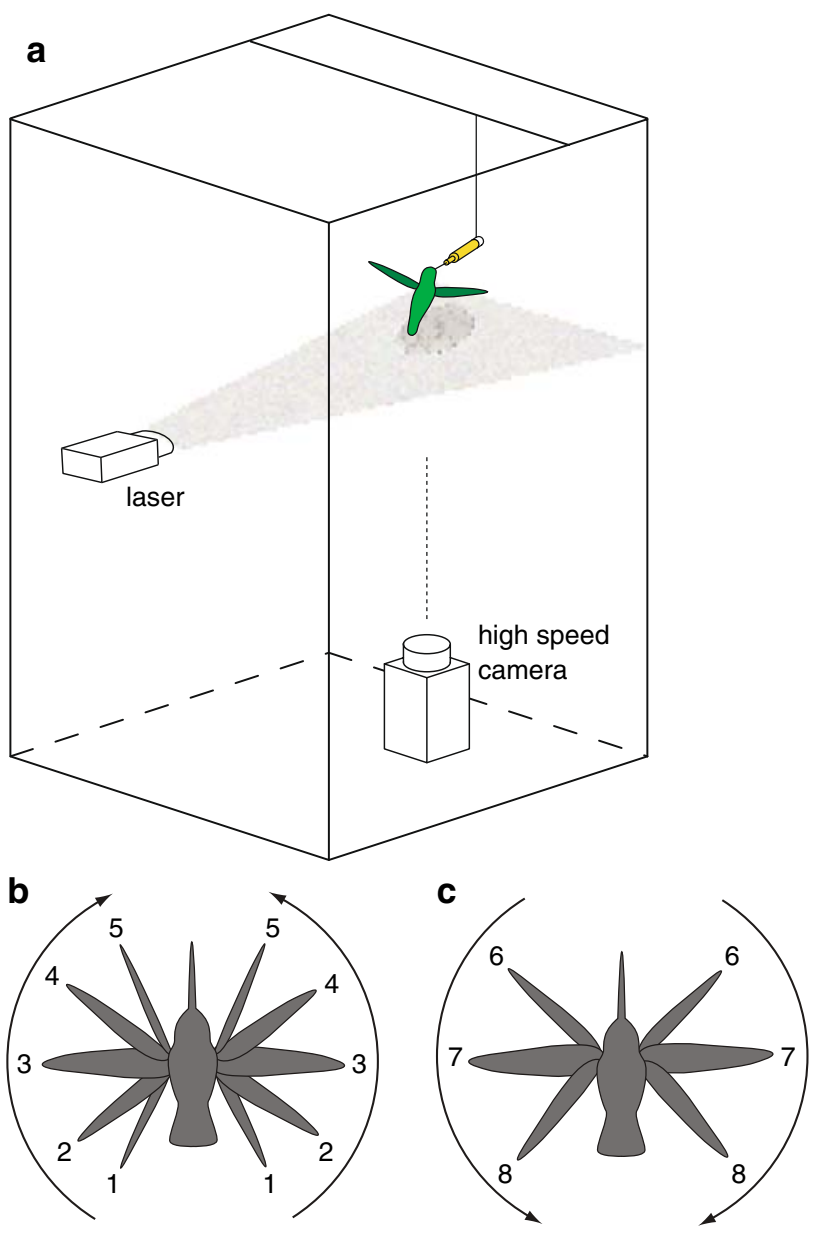

Fig. 2 a The experimental chamber used in hummingbird PIV experiments. During different sequences the laser sheet was moved to be either within the tail, at the tail tip, or underneath the tail (shown here). $\mathbf{b}$ The wings were visible in most of the raw images recorded from the camera located underneath the bird. The wing positions were assigned to different numbers indicating the phase within a complete wingbeat cycle. Position 1 corresponds to the wings in the rearward position in the middle of pronation. Positions 2-4 correspond to progressive movement through the downstroke, and position 5 corresponds to the forward position in the middle of supination. $\mathbf{c}$ Positions $6-8$ correspond to the wings moving progressively through the upstroke

with correct sequences and timing through a $2.66-\mathrm{GHz}$ dualprocessor workstation $\left(\right.$ Intel $^{\circledR} \mathrm{Xeon}^{\mathrm{TM}}$ ). The laser sheet was synchronized with a high-resolution $(1,600 \times 1,192$ pixel $)$ POWERVIEW 2M CCD camera (TSI Inc., model 630157) with a 50-mm f/1.8 Nikkor lens and an exposure time of $260 \mu$ s. Camera aperture was set to 5.6. A total of 15 image pairs per second $(15 \mathrm{~Hz})$ were captured. Time difference between the two images in a pair was optimized for the best PIV quality to $\Delta t=100 \mu \mathrm{s}$. A grid for the PIV processing was formed using the Nyquist Grid Engine over $64 \times 64$ pixels interrogation regions. Fast Fourier transform correlation was used with the Gaussian peak engine. 


\section{PIV flow field analysis}

We recorded at least ten hovering bouts for each of the hummingbirds, but limited our analysis to the 11 experiments during which the birds maintained constant body orientation through the trial. The instantaneous velocity fields and vorticity were calculated from all image pairs in this group of experiments. The flight chamber was sufficiently large that we could ignore wall influences to the flow because the change in lift per unit wing span due to the chamber walls (Katz and Plotkin 2001).

$\Delta L=\frac{\pi^{2}}{24} \frac{c^{2}}{h^{2}} \sim 0.01 \ll 1$,

where $h$ is chamber height, and $c$ is the wing span.

We further narrowed our analysis to the velocity measurements (i.e., image pairs) that could be grouped into one of eight wing positions within a complete wingbeat cycle (see Fig. 2b, c). This was accomplished by visual assignment of wing positions based upon each raw image. Arranging the measurements by wing position allowed us to compile a stroke sequence of velocity and vorticity profiles even though the images came from separate wingbeats. It is important to note that the wing tip velocity is greatest during mid-stroke and slowest during stroke transition (Fig. 1). Therefore the time difference between wing positions (average $\sim 3 \mathrm{~ms}$ ) was not constant.

The different experiments yielded different perspectives of the bird and wake both due to design and behavioral variability. In the former case, we moved the camera either closer to the feeder thereby focusing on the front (ventral) half of the stroke cycle or $\sim 10 \mathrm{~cm}$ further away from the feeder to focus on the back (dorsal) half of the stroke cycle. In the latter case, the bird fed at different angles relative to the feeder in the horizontal plane providing either a left or right bias. To facilitate comparisons among these multiple views, we calculated velocity over specific regions of interest (ROI) and along bird-centered axes. The ROIs were constrained to one of three positions: (1) underneath either the left or right wing on the front (ventral) side of the bird, including wing positions 3-7; (2) underneath either the left or the right wing on the back (dorsal) side of the bird, including wing positions 1-3 and 7-8; (3) underneath the tail in a narrow region that covered the full range of tail movement. The original velocity vector components in the $x$ and $y$ axes, $u$ and $v$, respectively, were rotated to along the bird component, $V_{\mathrm{s}}$ (positive from ventral to dorsal) and to a component that is perpendicular to the ventral-dorsal axis, $V_{\mathrm{n}}$ (positive when moving from the bird's lateral right to lateral left).

Within a filmed hovering bout, the velocities were averaged over all frames corresponding to the same wing position and the same ROIs were used for all frames.
Because the area of the ROIs differed across experiments, we restricted our analysis to within experiment results rather than combining experiments to generate average values.

On average, the measured flow field was $5 \mathrm{~cm}$ below the wing stroke plane. We assumed the vertical velocity to be $U_{\text {vert }} \sim 2 \mathrm{~m} / \mathrm{s}$ based upon measurements from similarly sized rufous hummingbirds, S. rufus (Warrick et al. 2005). Thus, it is estimated to require an average of $25 \mathrm{~ms}$ for the flow structures to be advected from the wing to the measuring plane. Because the wingbeat frequency is $\sim 40 \mathrm{~Hz}$ (corresponding to a wingbeat period of $25 \mathrm{~ms}$ ), the measured flow field should be lagging by the full cycle behind the actual wing stroke visible on the raw PIV image.

Another important reason why we avoided combining experiments for analytical purposes is that the vertical distance between the wings and the measuring plane-and thus the time lag-differed across experiments. Within each experiment, the hummingbirds maintained a constant body orientation and the time lag between wake generation and measurement is invariant. However, across experiments, hummingbirds used slightly different body angles, which should produce slight phase shifts in the wingbeat and PIV measurement cycles. Accordingly, we present results for individual experiments and then focus on the common features among experiments to develop a model of the wake of hovering hummingbirds.

\section{Results of flow measurements}

A representative trial with a perspective on the bird's ventral, right region is presented in Fig. 3. Across wing positions the along bird velocity component $V_{\mathrm{s}}$, in the wing domain, exhibits a clear pattern of flow reversal from ventral to dorsal. The average $V_{\mathrm{s}}$ value is close to 0 . The perpendicular to bird velocity component $V_{\mathrm{n}}$, in the wing domain, indicates net flow from lateral to medial because velocity moving from the lateral right to the lateral left is indicated with positive values.

We further calculated the velocity components in the wing domain for all sequences in which the wings could be identified while the birds maintained stable positions. The along bird velocity components in the wing domain for this set of experiments is given in Fig. 4. Three measuring planes are referred as: in tail (highest), tail tip (middle) and under tail (lowest). No unique pattern was observed across different experiments, but the hummingbird used different body angles in different experiments and the ROIs were not identical between any two trials. For most of the experiments there is a similar pattern for the same measuring plane. For the highest measurement plane, velocity maxima are observed for wing positions 4 
a

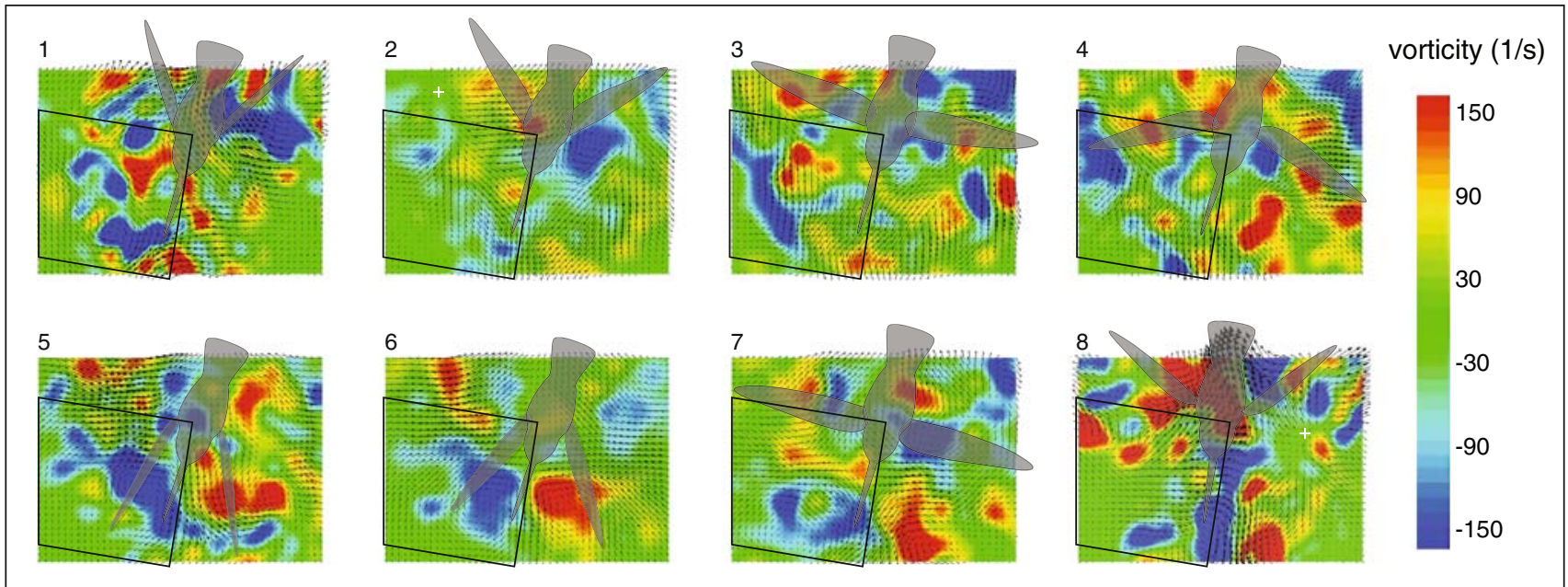

b

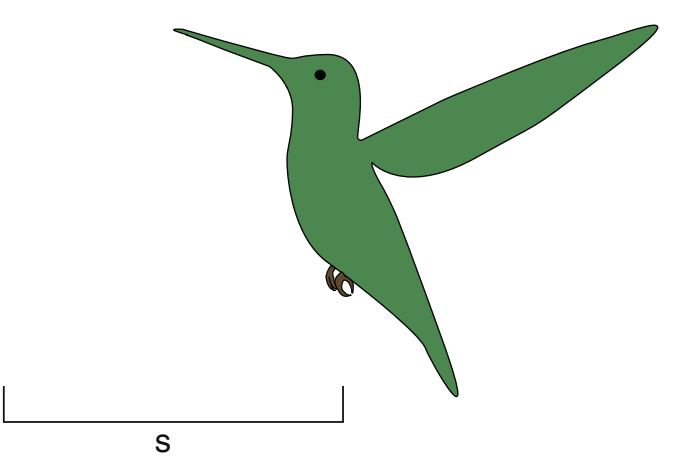

d

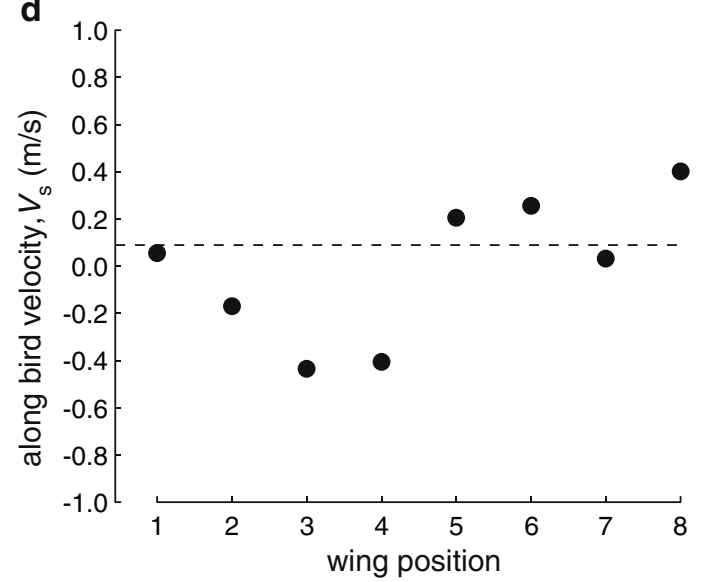

Fig. 3 Representative analysis of the wake underneath a wing, using experiment 5 as an example. a The raw images that contained the same wing position image were averaged to produce a color plot containing the vorticity (colors) and velocity vectors (arrows). The eight wing positions are numbered using the system described in Fig. 2b, c. Within each plot, a black polygon represents the region of interest over which velocity was calculated along the bird $\left(V_{\mathrm{s}}\right)$ and perpendicular to the bird $\left(V_{\mathrm{n}}\right)$. A small white cross indicates the center of the vortex loop if present. $\mathbf{b}$ The side view of an idealized hovering

5 , and minima are observed for positions $1-2$ and $7-8$. For the middle and lowest measurement planes, the overall pattern is shifted such that the velocity minima are
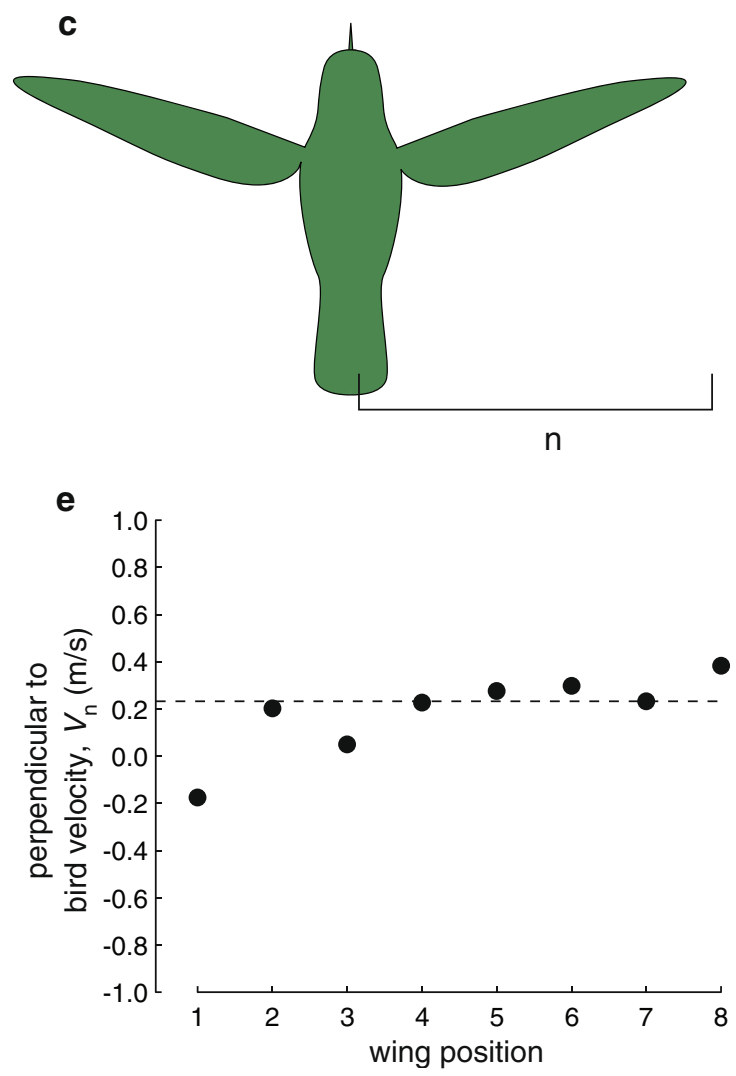

hummingbird, with the bottom of the black bracket indicating the planar region quantified in this dimension and the location of the laser sheet underneath the tail. $\mathbf{c}$ The backview of an idealized hummingbird, with the analogous perspective on the region of interest and level of the laser sheet. $\mathbf{d}$ The along bird velocity $\left(V_{\mathrm{s}}\right)$ across the eight wing positions. The dashed horizontal line indicates the mean velocity across all positions. e The perpendicular to bird velocity $\left(V_{\mathrm{n}}\right)$ across the eight wing positions

observed for wing positions 2-3. This apparent change in phase can be attributed to different distances between wing and measuring plane. Flow reversal, from head to 
in tail

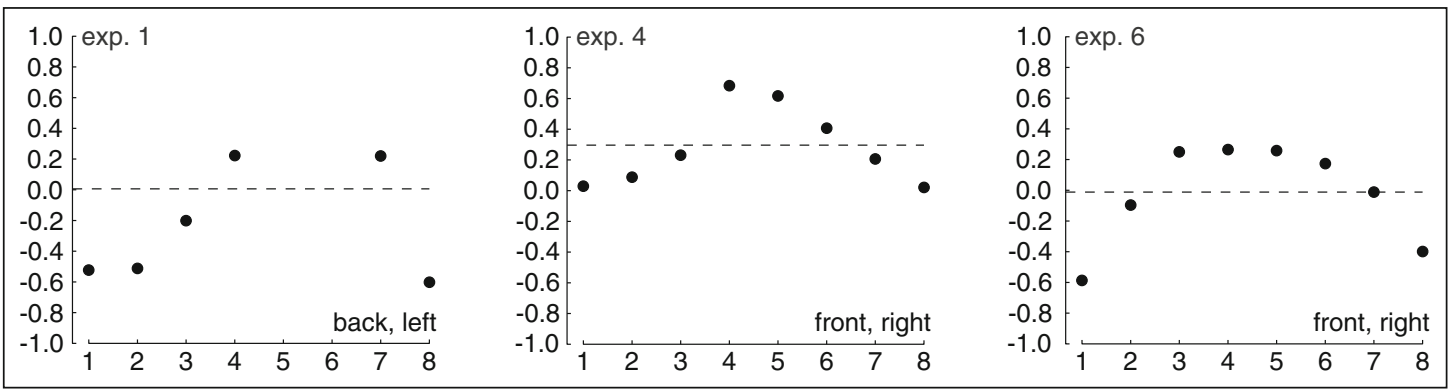

tail tip

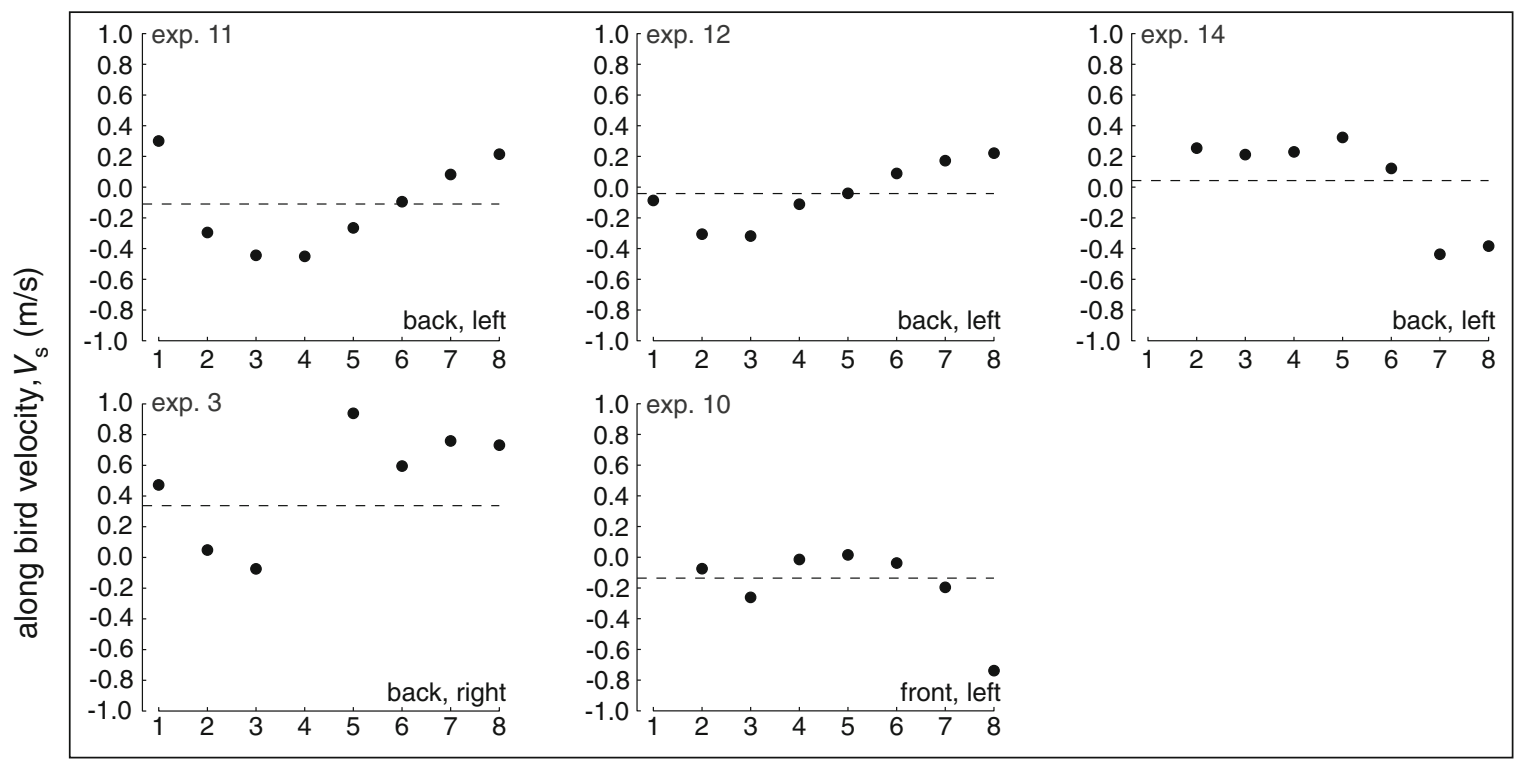

under tail

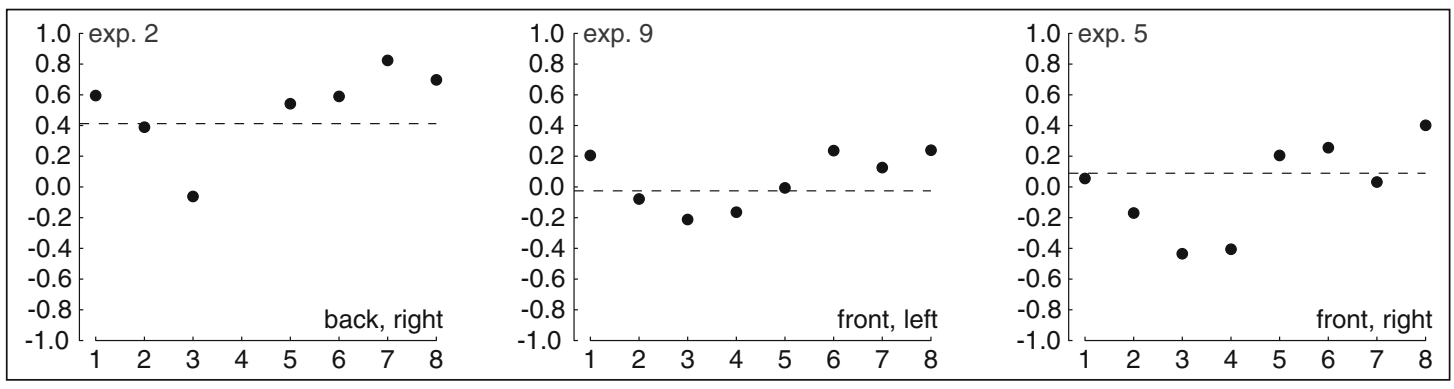

wing position

Fig. 4 Along bird velocity plots in the wing domain. The individual experiments are arranged first by tail position (grouping boxes), then by back to front, and lastly by left to right. Experiments $1-5$ come

tail and from tail to head, is observed in most but not all experiments.

The full experimental results for the normal to the bird velocity component, $V_{\mathrm{n}}$, in the wing domain are presented in Fig. 5. Throughout all experiments, velocity is from lateral to medial, i.e., the values are consistently negative for ROIs on the left side of the birds and consistently positive for ROIs on the right side of the birds. from bird 1. Experiment 6 comes from bird 2. Experiments 9-14 come from bird 3. The wing positions are indicated on the $x$-axis. The dashed line indicates the mean value across all wing positions

We further examined the subset of experiments that included complete views of the region around the tail oscillations. A representative sequence is provided in Fig. 6. The along bird velocities in the tail domain are presented in Fig. 7. Almost without exception, the velocities are positive across all wing positions, indicating that the net flow is in the ventral to dorsal direction for the region around the tail. In the majority of experiments in the 
in tail

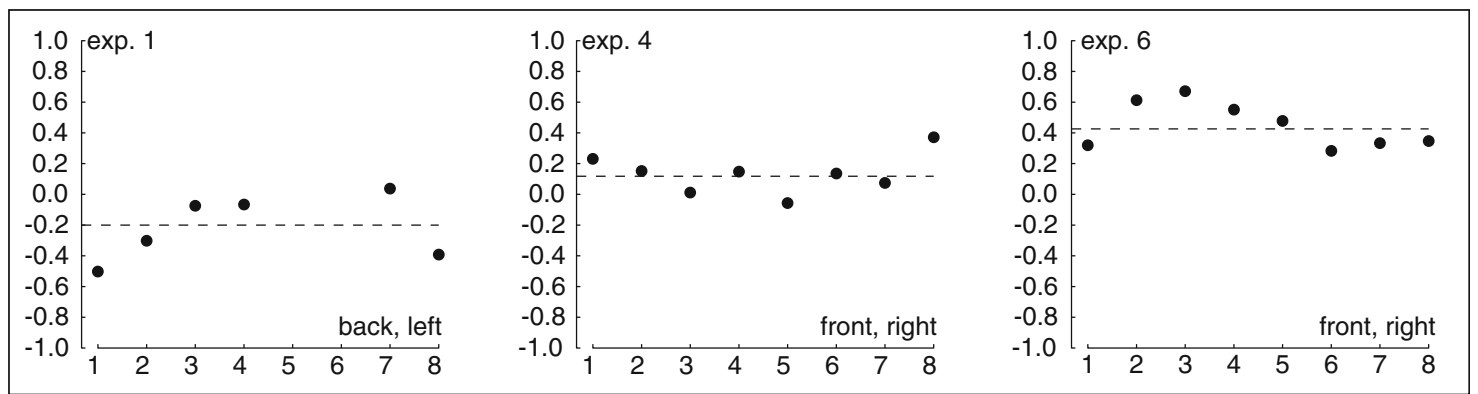

tail tip

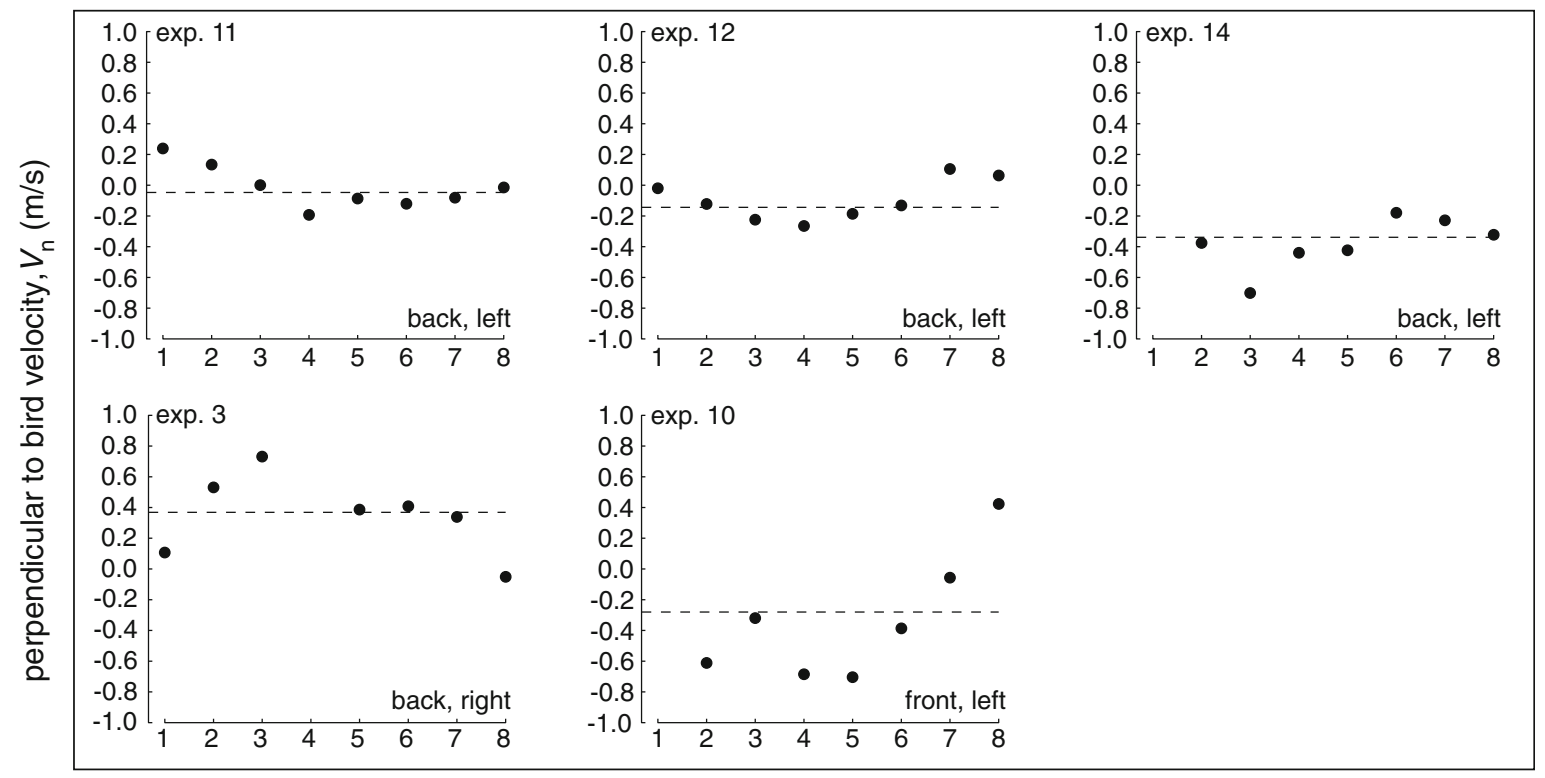

under tail

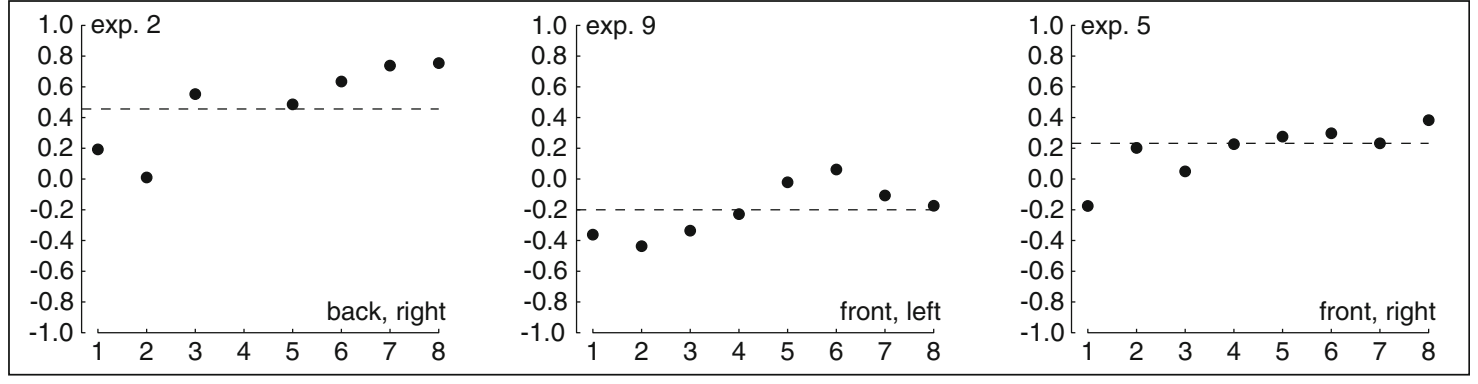

wing position

Fig. 5 Perpendicular to bird velocity plots in the wing domain. Note that all left perspectives have net negative velocities and all right perspectives have net positive velocities, indicating consistent flow from the periphery toward the bird. Details as in Fig. 4

upper and middle planes, the $V_{\mathrm{s}}$ velocity is minimal at positions 1-2 and 8. Taken together with our assumed lag of the wing wake, these data suggest that the bird is using its tail to deflect the outgoing flow caused by wings. In the lowest measuring plane for which we have only a single sample, this minimum is phase shifted.

In addition to measuring the net velocities with the ROIs, we also considered the mass flux over the entire measuring field to identify prominent sites with out of plane flow. These sites are indicated by discrete regions in which velocity emerges in all direction thereby suggesting the center of vortex ring or loop. In every experiment, we identified at least one, and usually two, wing positions in which there was evidence for vortex loops. Examples can be seen under the right wing for position 2 and under the left wing for position 8, in Fig. 3. A small white cross indicates the centers of these loops. For experiments in which both sides of the animal were visible, we identified 


\section{a}

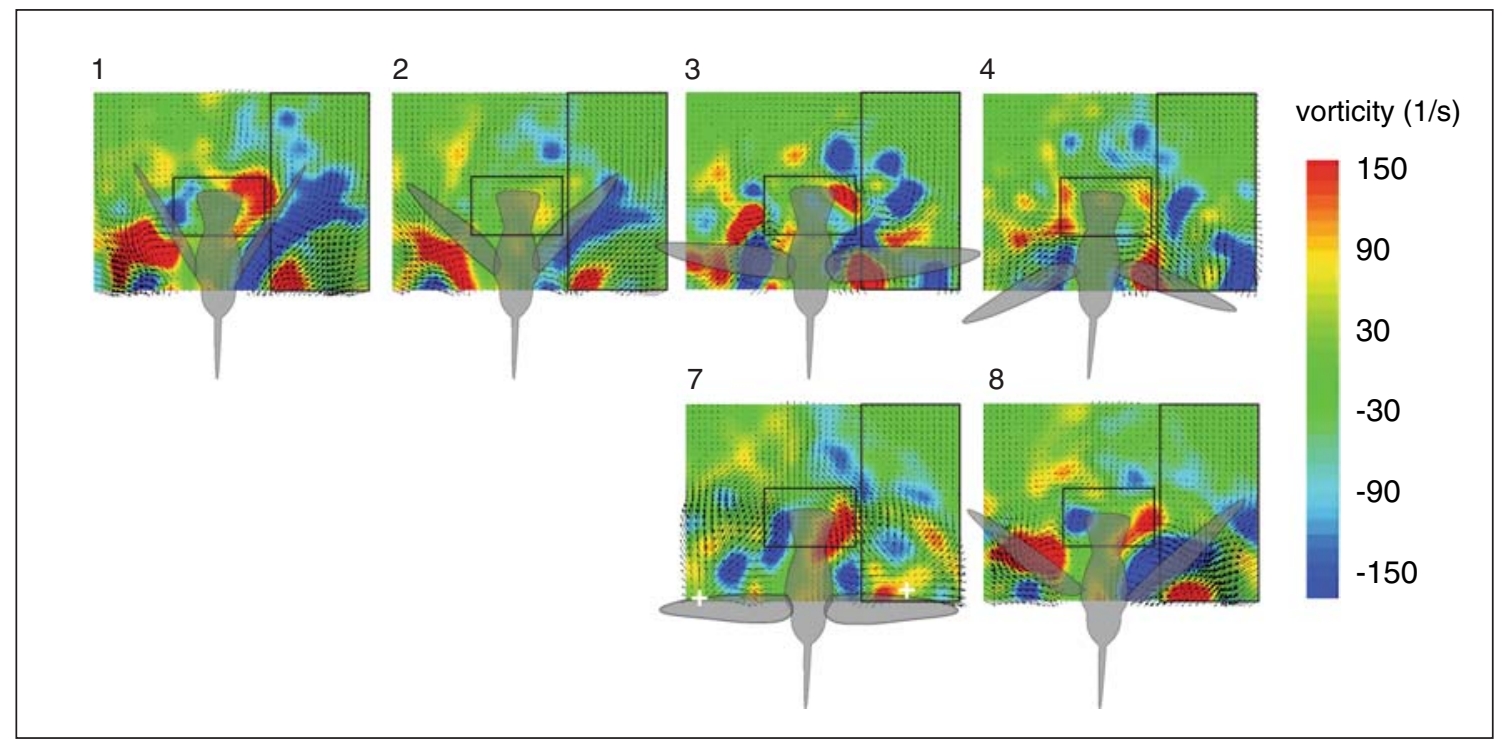

b

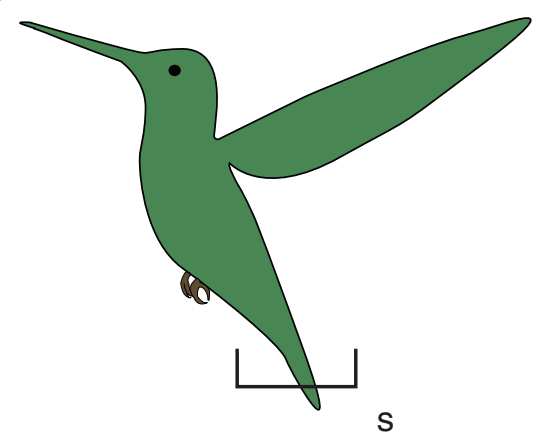

Fig. 6 Representative analysis of the wake underneath a tail, using experiment 1 as an example. a The color plots are configured using the same arrangement as in Fig. 3. However, these images were centered on the tail and wing positions 5 and 6 could not be identified because the both wings were out of view. Velocity was calculated only for the along bird $\left(V_{\mathrm{s}}\right)$ dimension. A small white cross indicates

separate vortex loops on either side of the animal. A coarse example of this pattern can be seen in wing position 7 in Fig. 6. In Fig. 8 we present a series of representative instantaneous velocity fields from two experiments that clearly indicate the existence of vortex loops that are present during both up- and down-strokes and that are underneath both wings.

\section{Discussion}

Measurements of the hovering hummingbird wake in the horizontal plane near the tail revealed three general patterns of flow velocity: (1) Along the ventral-dorsal axis underneath the wings, air flow switches direction more or

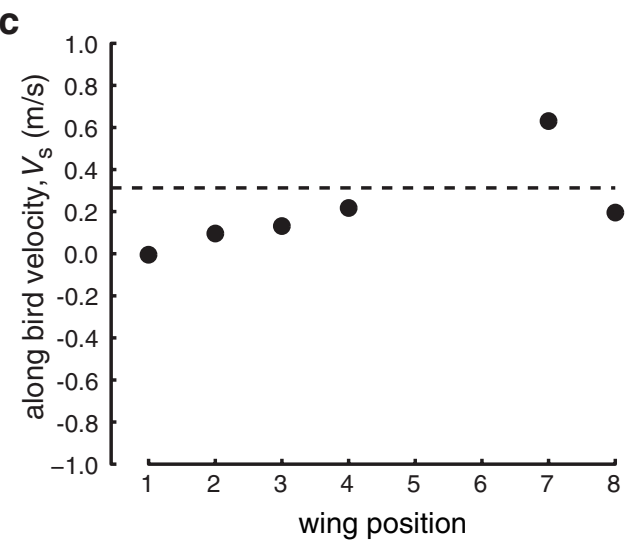

the center of the vortex loop if present. b The side view of an idealized hovering hummingbird, with the black bracket indicating the region quantified in this dimension and the location of the laser sheet underneath the tail. $\mathbf{c}$ The along bird velocity $\left(V_{\mathrm{s}}\right)$ across the six available wing positions. Details as in Fig. 3d, e

less sinusoidally indicating the influence of forwards (downstroke) and backwards (upstroke) movement of the wings (Fig. 4); (2) Perpendicular to this axis, underneath the wings, air flows from the periphery (lateral) in toward to the region underneath the hummingbird's body (medial) but with varying intensity (Fig. 5); (3) In a small region around the tail, the air flow is consistently oriented along the head-tail axis and away from the body (Fig. 7). In addition, we see clear evidence for separate vortex loops that are shed underneath each wing, and separate loops that are shed during down- and up-strokes.

As is the case for other PIV measurements from real flying animals, these flow fields reveal a complex wake that contains many disrupted structures and secondary flows. Furthermore, the patterns of flow reversal do not fit perfect 

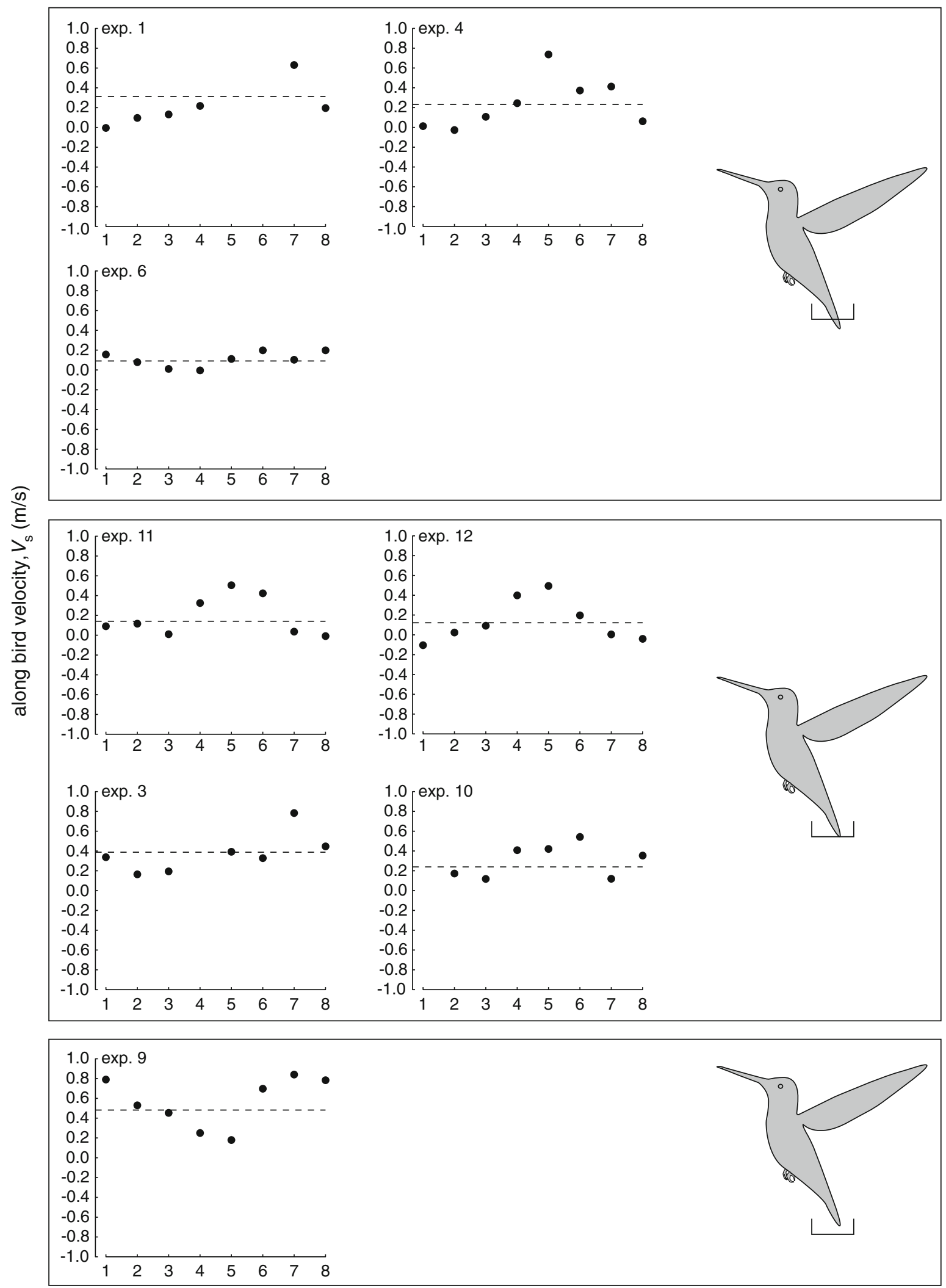

wing position

Fig. 7 Along bird velocity plots in the tail domain. Details as in Fig. 4 
Fig. 8 Examples of prominent vortex loops. The flow fields in experiment 6 revealed prominent vortex flow structures at bird wing positions 2 (a) and 7 (b), which presumably correspond to vortices shed during down- and up-strokes respectively. The flow fields from experiment 14 where particularly illustrative of separate flow structures underneath each wing at wing position $7(\mathbf{c})$. Note that these images come from single frame pairs and are not averaged across wing positions as was the case in previous flow field plots. The actual positions of the birds and their wing positions are shaded in translucent gray and the hypothesized vortex loops are marked by dashed lines. A small white cross indicates the center of each vortex loop
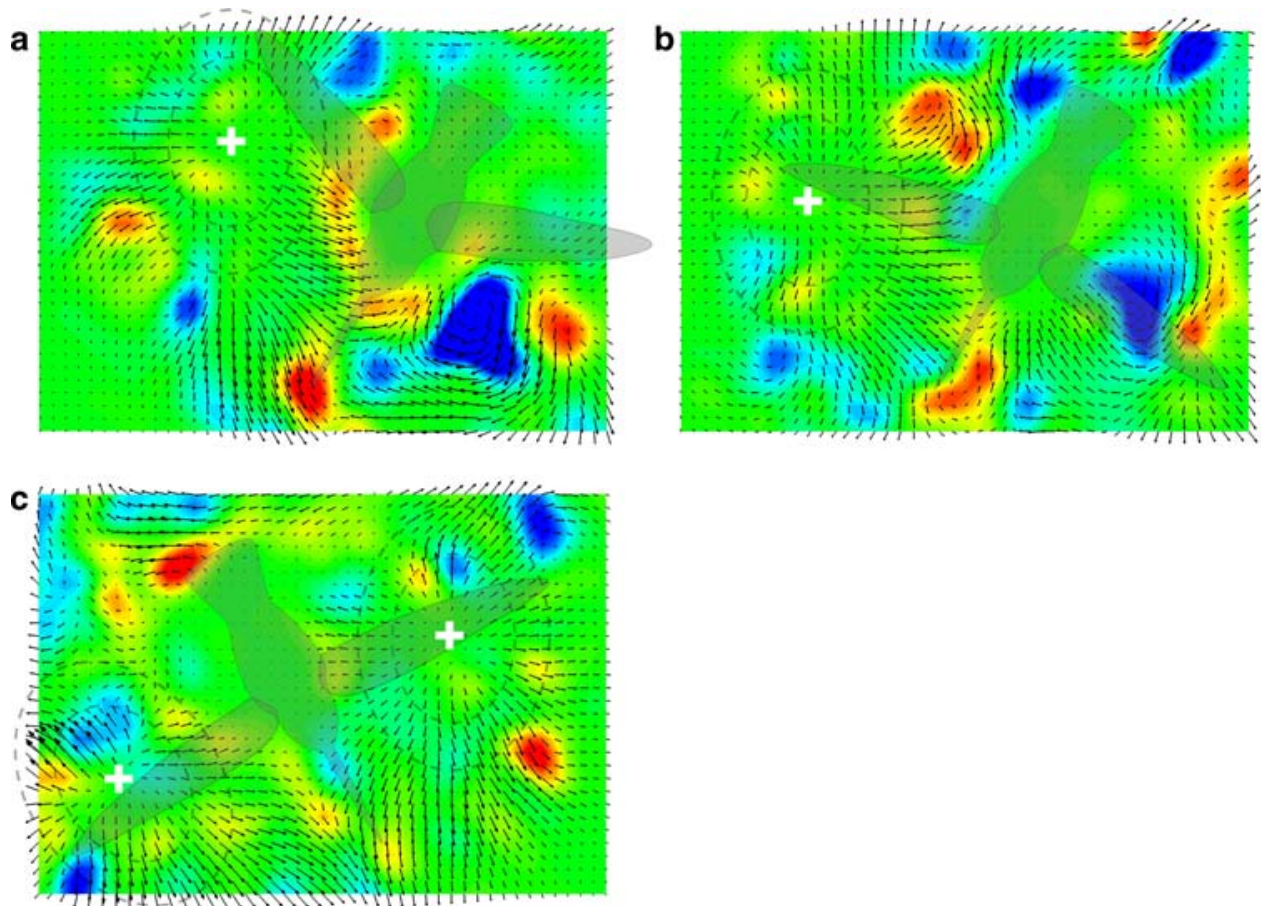

sinusoids as might be expected. However, it is unrealistic to expect invariant flow fields with perfect sinusoids because real hummingbirds exhibit differences in angle of attack, airfoil shape and stroke period between down- and up-strokes (Tobalske et al. 2007; Warrick et al. 2005; D.L. Altshuler, unpublished data). Additionally, we expect that the animals make rapid adjustments to these and other kinematic features to control stability, which will produce flow fields that vary across wingbeats.

It is not possible to infer the complete three-dimensional structure of the hummingbird's wake from the single perspective described here. Although there are relatively few measurements of the flow fields of different flying animals, we can compare our results with previous studies of forward flight in pigeons (Spedding et al. 1984), passerines (Hedenström et al. 2006; Rosén et al. 2007; Spedding et al. 2003) and bats (Hedenström et al. 2007; Muijres et al. 2008). In addition to these vertebrate fliers, the flow fields of numerous insect taxa have been measured during hovering and forward flight (Bomphrey et al. 2005, 2006; Brodsky 1991; Grodnitsky and Dudley 1996; Grodnitsky and Morozov 1992, 1993; Srygley and Thomas 2002; Willmott et al. 1997). Most of these invertebrate studies concerned single vertical perspectives oriented along the body axis and therefore do not provide information about the contributions of bilateral wings to overall flow field structures. However, Bomphrey et al. (2006) constructed a composite view from serial sections and concluded that the Tobacco Hawkmoth (Manduca sexta) uses its wings to produce a single vortex loop during downstroke in forward flight.

Wake descriptions from slow-flying bats and faster-flying birds differ in two fundamental aspects: (1) bat wings produce two vortex loops whereas birds produce a single vortex loop per stroke, and (2) bats do not exhibit strong circulation during stroke transitions unlike birds. Our data indicate that during hovering flight, hummingbirds more strongly resemble the bat pattern. First, we see clear evidence for separate vortex loops for each wing (Fig. 8). Second, our kinematic results indicate that wingtip velocities approach 0 at stroke transition whereas for other birds during forward flight, airstream velocity at the wing tips remains high. Because aerodynamic forces are proportional to the square of velocity, vortex generation should cease as the wings decelerate near stroke transition for hovering hummingbirds. It would be highly informative to consider the flow field patterns of hummingbirds across a wide range of flight speeds.

Combining the results from velocities and vorticity leads to a model for the principal flow structures in hovering hummingbirds (Fig. 9). The vortex loops shed during downstrokes move ventrally and loops shed during upstroke move dorsally. As air is sucked into these vortices, the principle mass comes from lateral to the bird because the bird's body acts as a boundary. Thus, the vortex loops move from lateral to medial.

With this model in mind, we can now consider the how the tail interacts with the wing wake. Although the tail 


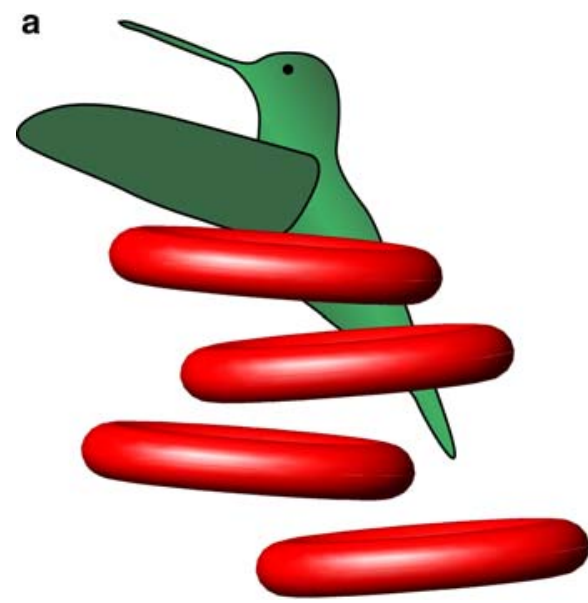

Fig. 9 Proposed model for the principal vortex wake structures of hovering Anna's hummingbirds, with left lateral (a) and dorsal (b) views. The vortex loops are elliptical in shape, being longer in the ventral-dorsal than the lateral-medial axis. Two prominent structures are produced during each wingbeat cycle when the wings reach

oscillates with an approximately sinusoidal motion, the velocities around the actual tail are either 0 or positive, indicating net flow along the body axis but away from the bird. These velocities have high magnitudes and are almost certainly not a consequence of the wake imparted by the tail motion. Instead, these flows are likely the result of wake encounter by the two wings coming toward each other during the end of the upstroke. We hypothesize that the tail is deflecting these larger flows, although the magnitude of interference with the wake will depend upon the degree of tail spreading and the precise phasing of tail oscillation. Because the magnitude of the pressure force will be proportional to the square of the velocity, the hummingbird should have the capability of generating tail moments of sufficient size to use this structure in maintaining pitch stability.

In this communication, we focus on the analysis of the mean flow patterns for different wing and tail positions. This was done using measured horizontal velocity components in the horizontal plane. Although it is our eventual goal, at this stage we are not attempting to explain the force balance of the hovering bird through momentum or vorticity analysis because such computations would have to involve doubtful assumptions pertaining to the full threedimensional flow field surrounding the bird. Dabiri (2005) demonstrated that the spatial velocity distribution in the wake is not sufficient for determination of locomotive forces but has to be combined with the pressure distribution. He proposed a method for accounting for the pressure contribution via wake vortex added-mass in analogy with the added-mass of fluid surrounding solid bodies.

At the present state, computational fluid dynamics and direct numerical simulation cannot be applied with

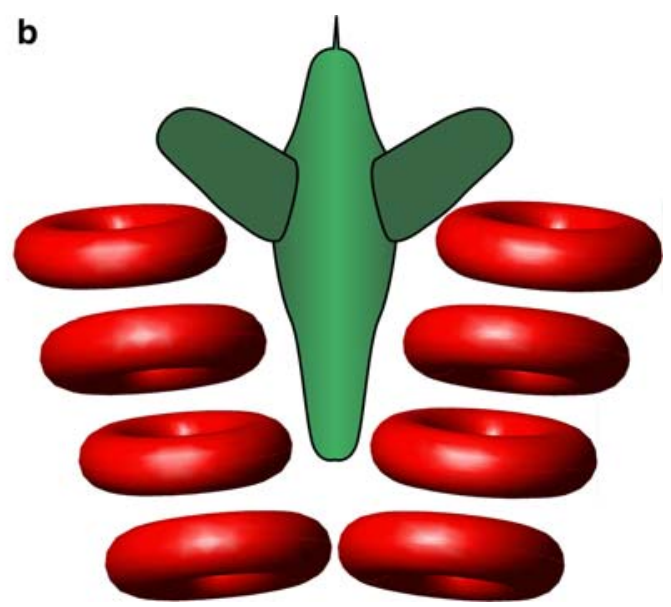

maximum velocity during the up- and down-strokes. The vortices shed during downstrokes move ventrally and vortices shed during upstrokes move dorsally. All vortex loops move from lateral to medial positions after shedding. Other, less prominent flow structures such as cross-stream vorticity are not illustrated

confidence to bird flight. To develop and validate numerical models and to obtain information needed for analytical models (e.g., information to quantify added-mass tensor component proposed by Dabiri 2005) more detailed measurements are required. New technologies for real threedimensional flow measurements should provide the necessary information, and can be combined with a numerical model to extract the pressure fields needed for full force balance analysis. In addition, such pressure estimates can be validated against existing, limited, pressure measurements (e.g., as in Usherwood et al. 2005). Particle tracking velocimetry can be applied directly to determine the Lagrangian properties of the flow, which can be used to determine the added-mass contributions (Dabiri 2005; Darwin 1953).

Acknowledgments We thank David Lentink for comments on an earlier version of the manuscript, and Bret Tobalske and Paolo Segre for suggestions for experimental procedures. We also gratefully acknowledge Christian Bartolome, Brian Cho, Sirish Makan, Elsa Quicazán, James Soh, and Kenneth Welch for assistance with data collection and analysis. This research was supported by a Regents Faculty Fellowship to Douglas L. Altshuler.

Open Access This article is distributed under the terms of the Creative Commons Attribution Noncommercial License which permits any noncommercial use, distribution, and reproduction in any medium, provided the original author(s) and source are credited.

\section{References}

Adrian RJ (1988) Review of particle image velocimetry research. In: The symposium on optical methods in flow and particle diagnostics, 6th international congress on applications of lasers 
and electro-optics, vol 9. Optics and Lasers in Engineering, San Diego, pp 317-319

Balmford A, Thomas ALR, Jones IL (1993) Aerodynamics and the evolution of long tails in birds. Nature 361:628-631

Bomphrey RJ, Lawson NJ, Harding NJ, Taylor GK, Thomas ALR (2005) The aerodynamics of Manduca sexta: digital particle image velocimetry analysis of the leading-edge vortex. J Exp Biol 208:1079-1094

Bomphrey RJ, Lawson NJ, Taylor GK, Thomas ALR (2006) Application of digital particle image velocimetry to insect aerodynamics: measurement of the leading-edge vortex and near wake of a Hawkmoth. Exp Fluids 40:546-554

Brodsky AK (1991) Vortex formation in the tethered flight of the peacock butterfly Inachis io L. (Lepidoptera, Nymphalidae) and some aspects of insect flight evolution. J Exp Biol 161:7795

Dabiri JO (2005) On the estimation of swimming and flying forces from wake measurements. J Exp Biol 208:3519-3532

Darwin C (1953) Note on hydrodynamics. Proc Camb Philos Soc 49:342-354

Evans MR, Hatchwell BJ (1992) An experimental study of male adornment in the scarlet-tufted malachite sunbird. II. The role of elongated tail in mate choice and experimental evidence for a handicap. Behav Ecol Sociobiol 29:421-427

Fry SN, Sayaman R, Dickinson MH (2003) The aerodynamics of freeflight maneuvers in Drosophila. Science 300:495-498

Grodnitsky DL, Dudley R (1996) Vortex visualization during free flight of Heliconiine butterflies (Lepidoptera: Nymphalidae). J Kans Entomol Soc 69:199-203

Grodnitsky DL, Morozov PP (1992) Flow visualization experiments on tethered flying green lacewings Chrysopa dasyptera. J Exp Biol 169:143-163

Grodnitsky DL, Morozov PP (1993) Vortex formation during tethered flight of functionally and morphologically two-winged insects, including evolutionary considerations on insect flight. J Exp Biol 182:11-40

Hedenström A, Rosén M, Spedding GR (2006) Vortex wakes generated by robins Erithacus rubecula during free flight in a wind tunnel. J R Soc Interface 3:263-276

Hedenström A, Johansson LC, Wolf M, von Busse R, Winter Y, Spedding GR (2007) Bat flight generates complex aerodynamic tracks. Science 316:894-897

Katz J, Plotkin A (2001) Low speed aerodynamics, 2nd edn. Cambridge University Press, Cambridge
Kokshaysky NV (1979) Tracing the wake of a flying bird. Nature 279:146-148

Møller AP (1989) Viability costs of male tail ornaments in a swallow. Nature 339:132-135

Muijres FT, Johansson LC, Barfield R, Wolf M, Spedding GR, Hedenström A (2008) Leading-edge vortex improves lift in slow-flying bats. Science 319:1250-1253

Norberg UM (1995) How a long tail and changes in mass and wing shape affect the cost for flight in animals. Funct Ecol 9:48-54

Rosén M, Spedding GR, Hedenström A (2007) Wake structure and wingbeat kinematics of a house martin Delichon urbica. J R Soc Interface 4:659-668

Spedding GR (1986) The wake of a jackdaw (Corvus monedula) in slow flight. J Exp Biol 125:287-307

Spedding GR (1987a) The wake of a kestrel (Falco tinnunculus) in flapping flight. J Exp Biol 127:59-78

Spedding GR (1987b) The wake of a kestrel (Falco tinnunculus) in gliding flight. J Exp Biol 127:45-57

Spedding GR, Rayner JMV, Pennycuick CJ (1984) Momentum and energy in the wake of a pigeon (Columba livia) in slow flight. J Exp Biol 111:81-102

Spedding GR, Rosén M, Hedenström A (2003) A family of vortex wakes generated by a thrush nightingale in free flight in a wind tunnel over its entire natural range of flight speeds. J Exp Biol 206:2313-2344

Srygley RB, Thomas ALR (2002) Unconventional lift-generating mechanisms in free-flying butterflies. Nature 420:660-664

Thomas ALR (1997) On the tails of birds. Bioscience 47:215-225

Thomas ALR, Balmford A (1995) How natural selection shapes birds' tails. Am Nat 146:848-868

Tobalske BW, Warrick DR, Clark CJ, Powers DR, Hedrick TL, Hyder GA, Biewener AA (2007) Three-dimensional kinematics of hummingbird flight. J Exp Biol 210:2368-2382

Usherwood JR, Hedrick TL, McGowan CP, Biewener AA (2005) Dynamic pressure maps for wings and tails of pigeons in slow, flapping flight, and their energetic implications. J Exp Biol 208:355-369

Warrick DR, Bundle MW, Dial KP (2002) Bird maneuvering flight: blurred bodies, clear heads. Integr Comp Biol 42:141-148

Warrick DR, Tobalske BW, Powers DR (2005) Aerodynamics of the hovering hummingbird. Nature 435:1094-1097

Willmott AP, Ellington CP, Thomas ALR (1997) Flow visualization and unsteady aerodynamics in the flight of the hawkmoth, Manduca sexta. Phil Trans R Soc Lond B 352:303-316 